\title{
Geant4 Simulation of Production and Interaction of Muons
}

\author{
A. G. Bogdanov, H. Burkhardt, V. N. Ivanchenko, S. R. Kelner, R. P. Kokoulin, M. Maire, A. M. Rybin, and L. Urban
}

\begin{abstract}
A set of models for Monte Carlo simulation of production and interaction of high energy muons is developed in the framework of the Geant4 toolkit. It describes the following physics processes: ionization of high energy muons with radiative corrections, bremsstrahlung, electron-positron pair production, muon induced nuclear reactions, gamma annihilation into muon pair, positron annihilation into muon pair, and into pion pair. These processes are essential for the LHC experiments, for the understanding of the background in underground detectors, for the simulation of effects related with high-energy muons in cosmic ray experiments and for the estimation of backgrounds in future colliders. The applicability area of the models extends to $1 \mathrm{PeV}$. The major use-cases are discussed.
\end{abstract}

Index Terms-Bremsstrahlung, mesons, modeling, Monte Carlo method, positron beam.

\section{INTRODUCTION}

$\mathbf{T}$ HIS work is devoted to the description of the simulation of high energy electromagnetic interaction of muons in the Geant 4 toolkit [1]. The interest in muon simulation is driven by the following circumstances:

- muons can be produced in electromagnetic or weak interactions and are a clean product of decays of $\mathrm{Z}, \mathrm{W}^{ \pm}$, and new heavy particles;

- muon detection is an important part of coming LHC experiments;

- a muon collider is one of the future options in HEP;

- muons of super-high energies (up to PeV) are a tool for the investigation of primary cosmic rays including neutrino astrophysics.

Geant 4 physics is implemented in terms of processes associated with a particle type [1]. Each process may include several models for different energy range of the incoming particle. The

Manuscript received November 30, 2004; revised December 4, 2005. This work was supported by ESA (TEC-EE) LOA 050/2004.

A. G. Bogdanov, S. R. Kelner, and R. P. Kokoulin are with the Moscow Engineering Physics Institute (MEPhI), Moscow 115409, Russia (e-mail: RPKokoulin@mephi.ru).

H. Burkhardt is with CERN, Geneva, CH-1211, Switzerland (e-mail: Helmut.Burkhardt@cern.ch).

V. N. Ivanchenko is with CERN, Geneva, CH-1211, Switzerland, on leave from Budker Institute for Nuclear Physics (BINP), Novosibirsk 630090, Russia (e-mail: Vladimir.Ivantchenko@cern.ch).

M. Maire is with Institut National de Physique Nucleaire et de Physique des Particules (LAPP), Annecy-le-vieux 74941, France (e-mail: michel.maire@lapp.in2p3.fr).

A. M. Rybin is with Institute for High Energy Physics (IHEP), Protvino 142281, Russia (e-mail: Andrei.Rubin@ihep.su).

L. Urban is with Research Institute for Particle and Nuclear Physics (RMKI), Budapest H-1525, Hungary (e-mail: urban@mail.cern.ch).

Digital Object Identifier 10.1109/TNS.2006.872633
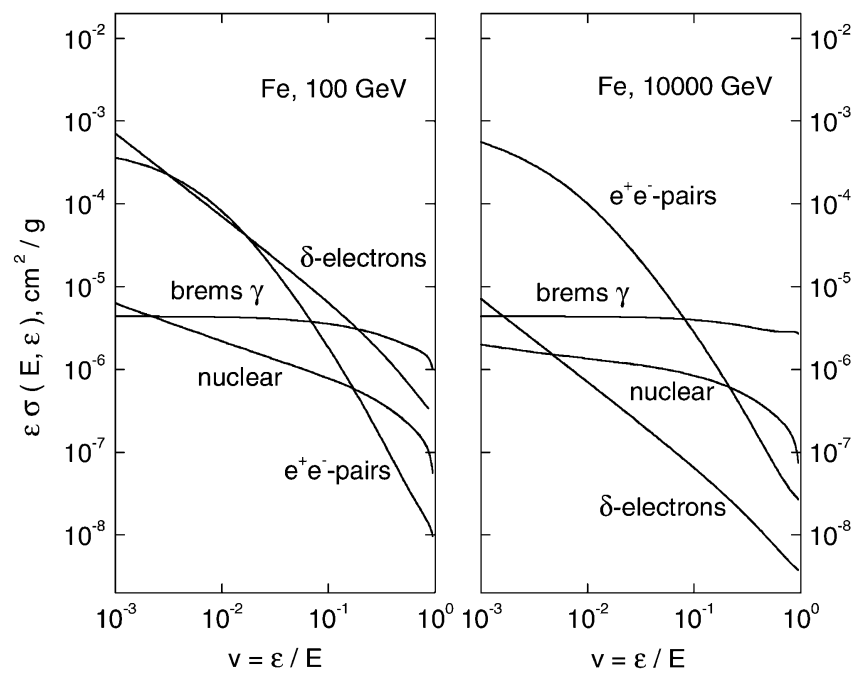

Fig. 1. Geant4 differential cross section for muon interaction processes in iron for production of $\delta$-electrons, $\mathrm{e}^{+} \mathrm{e}^{-}$pairs, bremsstrahlung $\gamma$, and hadrons.

set of processes and models for the simulation of muon interactions with matter will be discussed below. It is part of the Geant 4 Standard Electromagnetic Package [1]-[3].

\section{MUON INTERACTIONS}

There are four basic processes of muon interaction that determine muon energy loss and generation of secondary showers in matter: ionization (including production of high-energy $\delta$-electrons), production of electron-positron pairs, bremsstrahlung, and inelastic muon interaction with nuclei. Since the main contribution to the latter is given by low- $\mathrm{Q}^{2}$ region which is usually described in terms of nuclear absorption of virtual photons, it is often called "photonuclear" muon interaction.

\section{A. Cross Sections}

The dependence of macroscopic differential cross sections $\sigma(\mathrm{E}, \varepsilon)$ of these four processes in iron for two values of muon energy on the relative energy transfer $\mathrm{v}=\varepsilon / \mathrm{E}$ is shown in Fig. 1. E is the muon energy and $\varepsilon$ is the energy transfer. For convenient representation, the cross sections in the plot are multiplied by $\varepsilon$. The relative importance of a given process depends on the muon energy and atomic number $\mathrm{Z}$ of the material. Iron is chosen for demonstration of cross section shapes as a typical material for muon filters used in many HEP experiments.

At moderate muon energy, practically in the whole region the main interaction process is the production of $\delta$-electrons (in iron, $\mathrm{E}<100 \mathrm{GeV}$ ). At high energies, the relation between the cross sections of different processes is drastically changed. For 


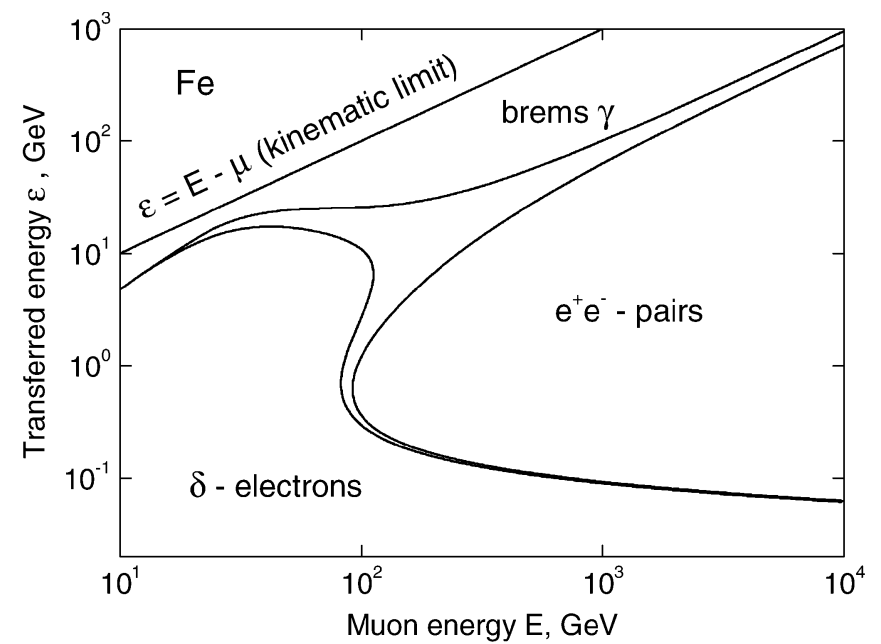

Fig. 2. Regions of dominance of different muon interaction processes in iron [4]. The curves correspond to $50 \%$ contribution of the process in the combined differential cross section.

example, for $\mathrm{E}=10 \mathrm{TeV}$ in a wide range of energy transfer the direct electron pair production dominates, for $\mathrm{v}=10^{-4}-10^{-2}$ its cross section being by 1-2 orders of magnitude greater than that of other interaction processes, and the relative contribution of the $\delta$-electron production becomes negligibly small.

Regions of dominance of different muon interaction processes are illustrated by the diagram presented in Fig. 2. The curves in the figure correspond to $50 \%$ contribution of the respective process to the combined differential cross section. For moderate muon energies, at any energy transfer (up to the limit defined by kinematics of $\mu$-e scattering) the most important process is the $\delta$-electron production. At higher energy transfer the process of muon bremsstrahlung dominates.

For high-energy muons $(\mathrm{E}>100 \mathrm{GeV})$, in a wide region of transfers up to $\mathrm{v} \approx 0.1$ the most frequent interaction process is the direct electron pair production. The contribution of photonuclear muon interaction to the combined cross section is always small (with the exception of lightest materials). However, this process leads to the production of nuclear showers, and it determines the main source of hadron background arising from high energy muon interactions.

\section{B. Muon Energy Loss}

Cross sections of muon bremsstrahlung, electron pair production by muons, and of inelastic muon interaction with nuclei nearly scale with the relative energy transfer

$$
\varepsilon \sigma(E, \varepsilon)=v \sigma(E, v) \approx f(v)
$$

Hence, the average muon energy loss related to each of the processes increases almost linearly with muon energy (Fig. 3). By analogy with the electrons, the combined contribution of these three processes to the total muon energy loss is often called "radiation loss". However, it should be noted that (unlike for electrons) the bremsstrahlung process is strongly suppressed because of a large muon mass. Contrary, the direct electron pair production plays an important role. Similar to the case of electrons, muon critical energy $E_{\mathrm{cr}}$ may be defined as the value at

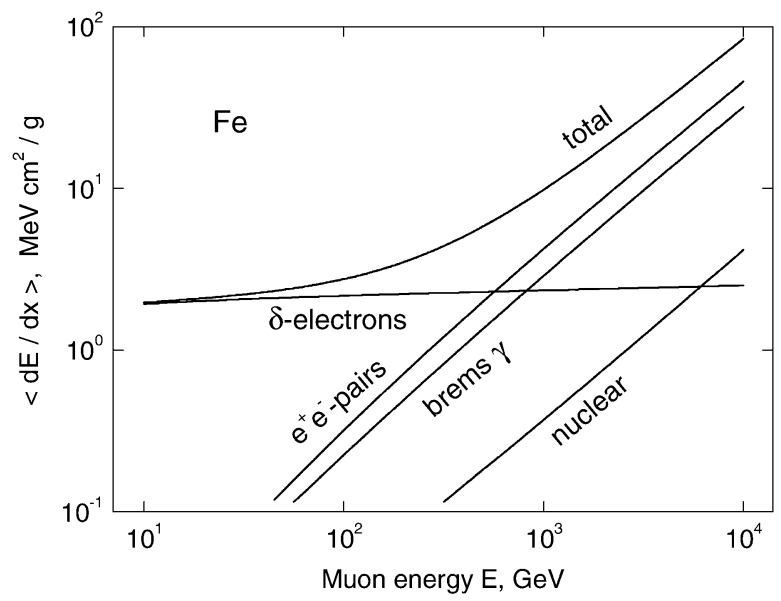

Fig. 3. Geant 4 calculation of different contributions to stopping power of muons in iron.

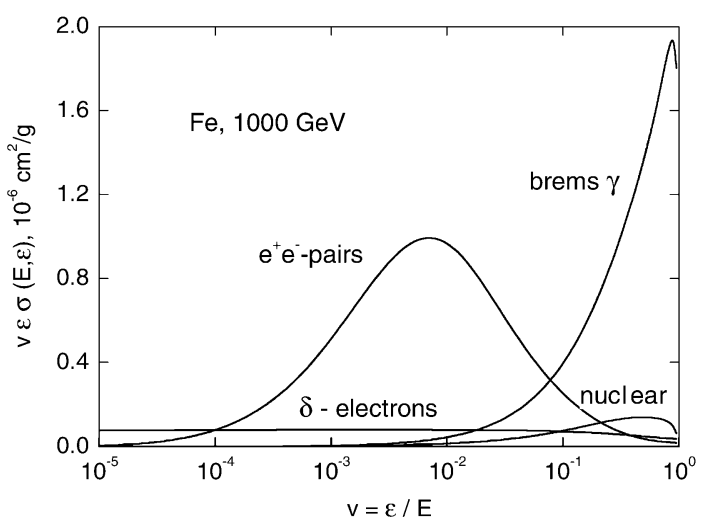

Fig. 4. Contribution of muon interaction processes in iron to the total energy loss as a function of the relative energy transfer in Geant 4 .

which the average ionization and radiation energy losses are equal [5]; for iron, $\mathrm{E}_{\mathrm{cr}} \approx 340 \mathrm{GeV}$.

The contribution of different energy transfer regions to the average energy loss is illustrated in Fig. 4. Since

$$
\begin{aligned}
\frac{d E}{d x} & =-\int_{0}^{\varepsilon_{m}} \varepsilon \sigma(E, \varepsilon) d \varepsilon \\
& =-E \int_{0}^{\varepsilon_{m}} \nu \varepsilon \sigma(E, \varepsilon) d(\ln \nu),
\end{aligned}
$$

in coordinates of Fig. 4 the contribution of the given process to the average specific energy loss is proportional to the area under the corresponding curve. Here $\varepsilon_{\mathrm{m}}$ is the maximum energy transfer. For electron pair production, the relative energy transfers contributing to the average energy loss are mostly those between $10^{-3}-10^{-1}$; for bremsstrahlung and inelastic interaction, the average loss is determined mainly by rare catastrophic collisions with $\mathrm{v}>0.1$. Energy loss for ionization is distributed almost uniformly in $\ln (\varepsilon)$ between $\ln (\mathrm{I})$ and $\ln (\mathrm{E})$, where $\mathrm{I}$ is the mean ionization potential.

\section{Models for Muon Processes}

Four Geant 4 physics processes are created for simulation of muon interactions: 
- G4MuIonization;

- G4MuBremsstrahlung;

- G4MuPairProduction;

- G4MuNuclearInteraction.

As pointed out above, the ionization process provides the main contribution to the muon energy loss for relatively low energies. The three following models are used for the different energy ranges:

- for $\mathrm{E}<0.2 \mathrm{MeV}$, muon energy losses are derived from the tabulated proton energy loss using scaling relation for the stopping power of heavy particles [6], which is a function only of the particle velocity;

- for $0.2 \mathrm{MeV}<\mathrm{E}<1 \mathrm{GeV}$, the Bethe-Bloch formula with shell and density corrections is applied [6];

- for $\mathrm{E}>1 \mathrm{GeV}$, the leading term of radiative corrections to the Bethe-Bloch formula is taken into account according to [7].

The fluctuations of ionization energy loss are simulated using the universal model [8]. In the limit of thick absorber (large step of muon in a media) it represents Gaussian Bohr fluctuations [6] and for thin absorber (small step) samples Landau type fluctuations.

In Geant4, for the description of muon bremsstrahlung the results of [9] are used, which represents an improved parameterization of the Petrukhin-Shestakov formula [10], and is valid for any degree of screening and accounts for nuclear form-factor. The formulae for muon bremsstrahlung on atomic electrons are taken from [7]. Bremsstrahlung from target electrons (together with respective radiative correction to $\mu$-e scattering) is taken into account as a correction term [7] to the Bhabha formula for $\delta$-electron production. The formulae from [11] and [12] describe electron pair production on a screened nucleus, while for the contribution of pair production on electrons the results [13] are used. For the cross section of inelastic muon interaction with nuclei, the expression from [14] is applied with VDM-based correction for nuclear shadowing [15] and energy dependent approximation of photonuclear cross section $\sigma_{\gamma \mathrm{N}}(\varepsilon)$ for real photons[16].

\section{Geant4 Implementation of Muon Interactions}

Energy loss processes in Geant4 have continuous and discrete components [1]-[3]. High energy transfers of energy are simulated as a real discrete act of an interaction. Low energy transfers are treated as a continuous process of energy loss. The mean value of this restricted energy loss is obtained as a result of integration (2). As the upper limit of the integral is used $\varepsilon_{m}=\min \left(\mathrm{T}_{\text {cut }}, \mathrm{T}_{\max }\right)$, where $\mathrm{T}_{\text {cut }}$ is Geant 4 production threshold for $\delta$-electrons, $\mathrm{T}_{\max }$ is the kinematical limit for a given process. Thus, energy transfers below $\varepsilon_{\text {lim }}$ are considered as continues energy loss. The same integration is applied to the processes of ionization, bremsstrahlung, and $\mathrm{e}^{+} \mathrm{e}^{-}$pair production. The sum of these three contributions is calculated at the initialization stage of Geant4 and stored in the form of the $\mathrm{dE} / \mathrm{dx}$ table [1]. At initialization consecutively to that, the range and inverse range tables are also calculated.
Additionally, the restricted cross section of each process is calculated and stored in the separate lambda tables

$$
\lambda(E)=\int_{\varepsilon_{m}}^{E} \sigma(E, \varepsilon) d \varepsilon
$$

where $\lambda(\mathrm{E})$ is the inverse interaction length, $\varepsilon_{\mathrm{m}}$ is the same threshold value as one used in the integration (2). Since the muon-nuclear interaction does not contribute significantly to the energy loss of muons (Fig. 3), this process is always treated as a discrete ones, and the energy threshold $\varepsilon_{\mathrm{m}}$ for this process is set to zero.

For all processes the differential cross section $\sigma(\mathrm{E}, \varepsilon)$ is used both for calculation of the cross section and for sampling of a final state. To save CPU during tracking time, the restricted cross sections $\sigma_{\mathrm{R}}(\mathrm{E}, \varepsilon, \mathrm{Z})$ are calculated at initialization for the processes of bremsstrahlung and $\mathrm{e}^{+} \mathrm{e}^{-}$pair production

$$
\sigma_{R}(E, \varepsilon, Z)=\int_{\varepsilon_{m}}^{\varepsilon} \sigma(E, x, Z) d x .
$$

These calculations are performed with uniform grid in logarithmical scale both for $\mathrm{E}$ and $\varepsilon$ for five different atomic numbers $\mathrm{Z}=1,4,13,29,92$, and this table is kept for further interpolation.

During tracking at each step the mean restricted energy loss of a muon is a calculated using stored table [1], then using this value the final continuous energy loss is sampled by applying the fluctuation model [8]. If one of the muon processes limited the step, then the sampling of the secondary particles is performed for this process at the end-point of the step. First, the atomic number $\mathrm{Z}$ is sampled using the atomic densities of the material and cross sections (4) obtained from the table. Secondly, using the same table the energy transfer is sampled. At the last step, the kinematical parameters of final particles are sampled. For the process of $\delta$-electrons the interaction with the nucleus is neglected-the electron is assumed to be free, so its scattering angle is defined by the energy transfer. For the bremsstrahlung process the angular distribution of gamma is sampled according to a simplified probability function

$$
f(x) \sim \frac{x}{\left(1+x^{2}\right)^{2}}, \quad x=\frac{E \theta_{\gamma}}{m}
$$

where $\theta_{\gamma}$ is the polar angle of the gamma, $m$ is the muon mass. For the process of $\mathrm{e}^{+} \mathrm{e}^{-}$pair production the muon deflection is neglected. The momentum of nuclear recoil is also neglected for the processes of ionization and bremsstrahlung. The energy-momentum balance is used to determine four-momentum of the scattered muon. By default, no production thresholds are applied in these two processes.

The muon photonuclear interaction is always treated as a discrete process-no contribution to the continuous energy loss (2). The total cross section (3) is obtained by the numerical integration of the expression from [14]. The process is considered for muon energies from $1 \mathrm{GeV}$ to $1000 \mathrm{PeV}$. The main contribution to the total cross section comes from relatively low four-momentum transfer $\left(\mathrm{Q}^{2}<1 \mathrm{GeV}^{2}\right)$, thus t-dependence of the differential cross section from [14] is used. The 


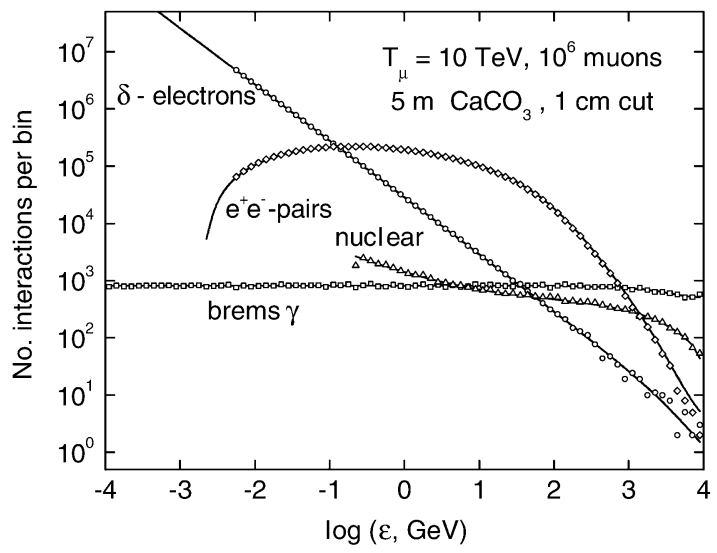

Fig. 5. Distribution of energy transfer for $10 \mathrm{TeV}$ muon interactions in five meters of $\mathrm{CaCO}_{3}$ : points-Geant $4.2 \mathrm{p} 01$, lines-theory. The production threshold $1 \mathrm{~cm}$ for secondary gamma, electron, and positron is used.

muon recoil is defined by the four-momentum balance. From the four-momentum transfer the hadronic vertex is sampled using Geant 4 parameterized hadronic model [1]. For the effective simulation of deep penetration of muons, there is the regime of the process, when hadronic vertex is not sampled at all—only muon four-momentum transfer is sampled.

\section{E. Validation and Discussion}

For an adequate description of muon transport in matter and of the response of the experimental setup, an accurate reproduction of the total and differential cross sections is of crucial importance.

Since there are many intermediate calculations and interpolations of the theoretical formula, the first step of validation of the implementation of the described models and processes is done by means of comparisons of the theoretical differential cross sections with the Geant 4 simulation results. A new Geant 4 extended example "MuonProcesses" has been designed for the demonstration of muon physics and for the validation of Geant 4 models. The idea of this test is the following: if a muon energy loss is $\Delta \mathrm{E}$ in a layer of some material and it is much less than the muon energy $\mathrm{E}$, then the latter may be considered unchanged, and individual interactions of the muon along its path are independent. In these conditions, distribution of the number of interactions $\mathrm{N}$ in the energy transfer may be readily written as

$$
d N / d(\ln \varepsilon)=N_{\mu} X \varepsilon \sigma(E, \varepsilon)
$$

where $\sigma(\mathrm{E}, \varepsilon)$ is macroscopic differential cross section in mass units $\left(\mathrm{cm}^{2} / \mathrm{g}\right), \mathrm{X}$ is the layer thickness (in $\left.\mathrm{g} / \mathrm{cm}^{2}\right)$, and $\mathrm{N}_{\mu}$ is the total number of muons. Results of the comparison of Geant 4 simulation predictions with theory calculations for $10 \mathrm{TeV}$ muons are shown in Fig. 5. Noteworthy, such a procedure allows checking not only the correctness of the description of the distribution shape of sampled energy transfers, but also the absolute value of the total cross section. It was found that in the energy range of model applicability the maximum systematic difference in the differential distribution does not exceed 5\% for bremsstrahlung and pair production, and is less than $1 \%$ for the $\delta$-electron process.
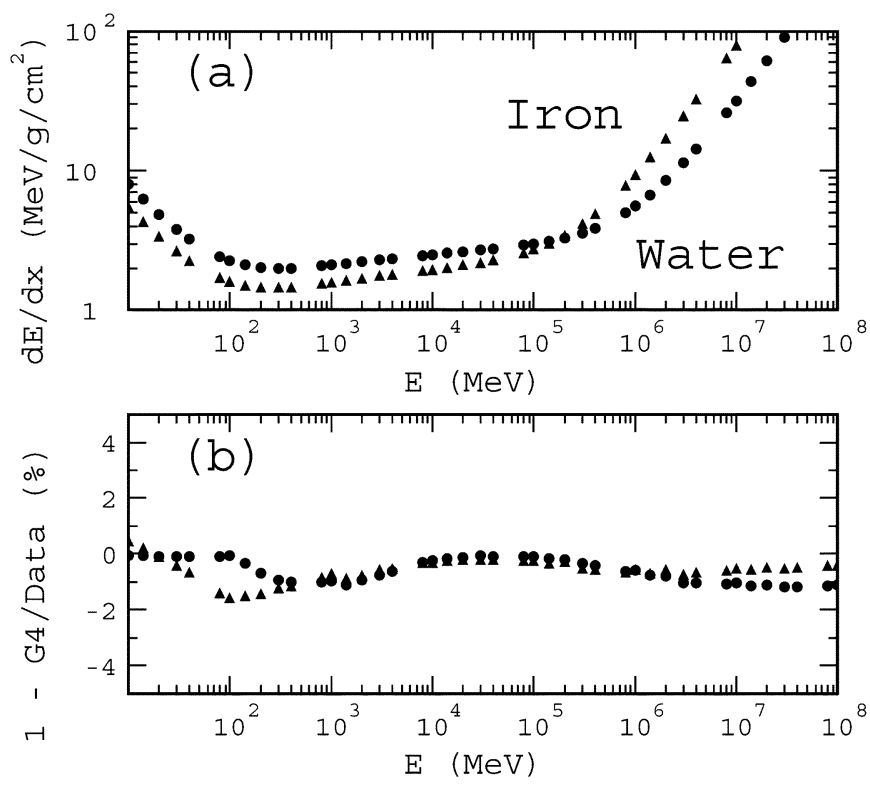

Fig. 6. Results of comparison of the muon stopping power between Geant 4 predictions and the evaluated data [5]: (a) stopping power of water (circles) and iron (triangles)-Geant 4 results and the data cannot be distinguished in the plot; (b) relative difference between Geant 4 predictions and the data.

As a whole, the accuracy of theoretical formulae used in Geant 4 for ultra-relativistic muons may be estimated as $1 \%-2 \%$ both for the differential cross section and for the average energy loss for muon energies from several $\mathrm{GeV}$ to (at least) several $\mathrm{PeV}$. At higher energies (depending on the material), the LPM suppression for electron pair production may appear important. Another exception is represented by heavy materials, where so-called Coulomb correction proportional to $(\alpha \mathrm{Z})^{2}$, again essential for direct electron pair production, is not taken into account. At worst, this may lead to an overestimation of the cross section by a few percent.

This set of theoretical formulae implemented for Geant 4 is nearly the same as the one used in recent calculations of muon energy loss and ranges in various materials [5]. The only exception is the cross section of inelastic interaction with nuclei, where the authors of [5] have taken results from [17]. However, as shown in [18], the difference between the two versions of the cross section for this process is determined mainly by a different choice of the cross section $\sigma_{\gamma \mathrm{N}}$ for real photons; with the same dependence $\sigma_{\gamma \mathrm{N}}(\varepsilon)$ formulae of [14] and [17] give similar (within $10 \%$ in the differential cross section and $5 \%$ for the average loss) results. Taking into account that in most materials the relative contribution of nuclear interaction is less than $10 \%$, this difference does not introduce a serious additional uncertainty.

In order to perform an independent verification of Geant 4 implementation of muon processes, a direct comparison of stopping power between evaluated data [5] and simulation results was performed. In this simulation production cuts were set to infinite and the sum of the mean energy loss of muons from ionization, bremsstrahlung, and pair production was calculated. The results of the comparison (Fig. 6) demonstrate the good agreement for any muon energy. The maximum difference does not exceed $2 \%$. 


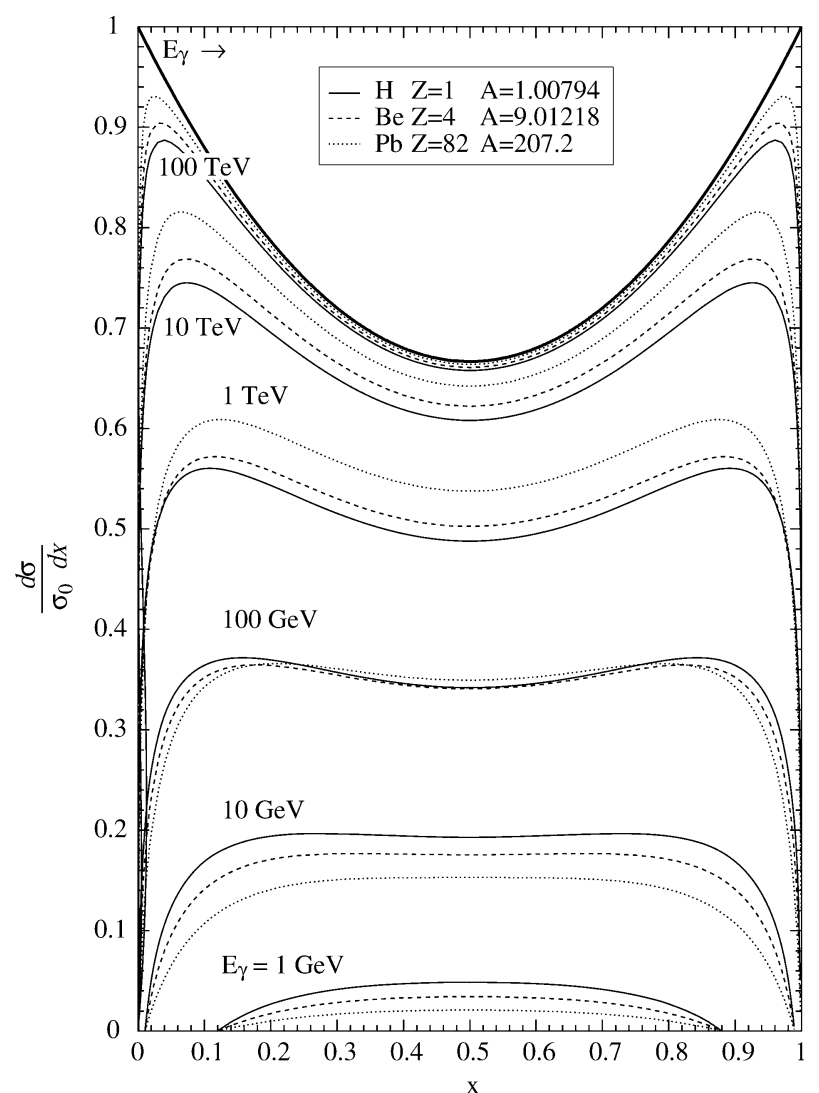

Fig. 7. Normalized differential cross section for muon pair production as a function of $\mathrm{x}=\mathrm{E}_{\mu} / \mathrm{E}_{\gamma}$, the energy fraction carried by one of the muons in the pair.

\section{Muon Production Processes}

A set of high energy processes of muon production is being developed inside the Geant 4 Standard Electromagnetic Package. The cross sections of these processes are relatively small. However, these processes may provide a serious background for high energy experiments.

\section{A. Muon Pair Production}

One of the new processes is gamma conversion to muon pair in the electric field of a nucleus. The cross section of this process can be expressed in terms of the cross section value $\sigma_{0}$

$$
\sigma_{0}=4 \alpha Z^{2} r_{\mu}^{2} \log \left(119 \cdot \frac{Z^{-1 / 3}}{A^{0.27}} \cdot \frac{m_{\mu}}{m_{e}}\right)
$$

where $\alpha$ is the electromagnetic coupling constant, $\mathrm{r}_{\mu}$ is the classical muon radius, $\mathrm{Z}$ and $\mathrm{A}$ are the nucleon charge and mass number, and $\mathrm{m}_{\mu}$ and $\mathrm{m}_{\mathrm{e}}$ are the electron and muon mass respectively. The cross section is proportional to $\mathrm{Z}^{2}$. There is also a weaker logarithmic dependence on $\mathrm{Z}$ and $\mathrm{A}$. A slightly different logarithmic term is used for hydrogen, see [19]. The differential cross section is shown in Fig. 7 in units of $\sigma_{0}$.

The material dependence of the differential cross section is not too strong and the cross section increases very slowly with energy toward an asymptotic limit, which corresponds to the complete screening. As it can be seen from Fig. 7, the asymptotic cross section of the process in the limit of high energy is $7 \sigma_{0} / 9$. The details of theory and implementation are described

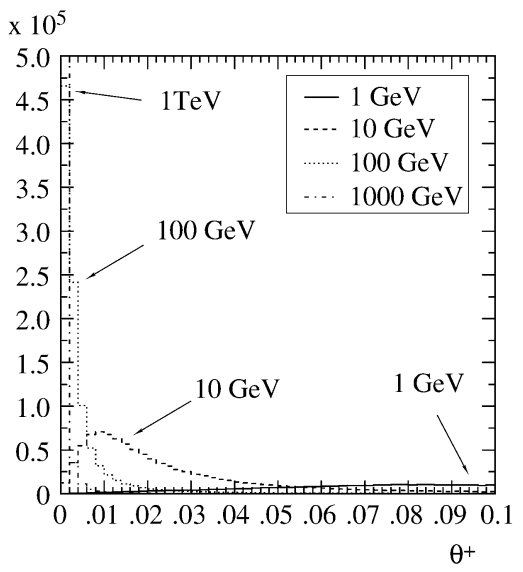

Fig. 8. Production angles of muons for different energies in carbon as observed in the laboratory frame sampled by Geant 4 .

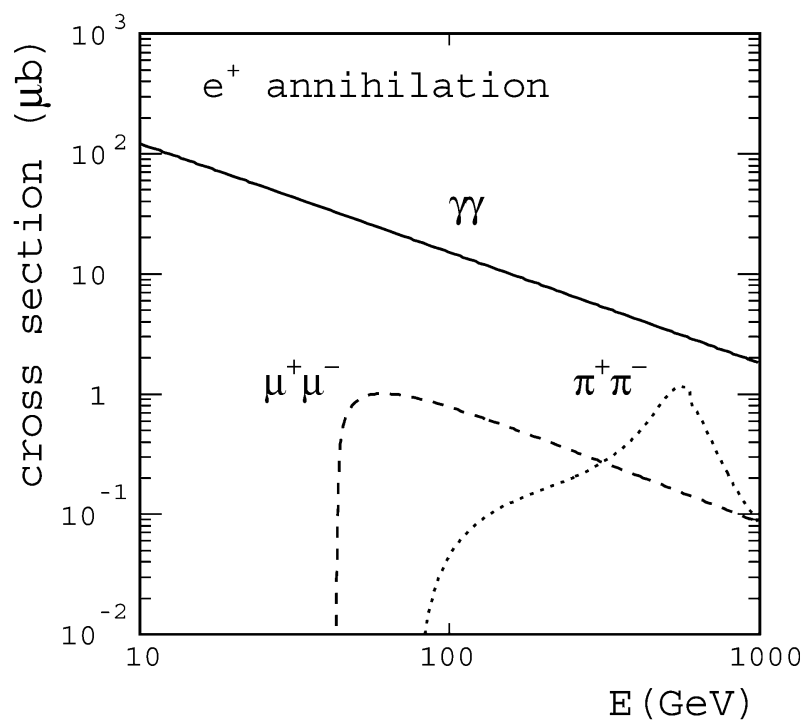

Fig. 9. Geant 4 cross sections per media electron of positron annihilation into two gamma, $\mu^{+} \mu^{-}$, and $\pi^{+} \pi^{-}$.

in [19]. The distributions of the laboratory angles of muons for this process are plotted in Fig. 8.

The Geant 4 class G4GammaConversionToMuons has been implemented. Through this process, muon pairs can be produced by high energy photons in dense materials of LHC detectors or in beam elements of a linear collider.

\section{B. Positron Annihilation to Muon Pair}

The annihilation of positrons with atomic electrons into muon pairs [20] requires a minimum energy of the positron of

$$
\mathrm{E}_{\mathrm{th}}=2 \mathrm{~m}_{\mu}^{2} / \mathrm{m}_{\mathrm{e}}-\mathrm{m}_{\mathrm{e}}=43.69 \mathrm{GeV} \text {. }
$$

The motion of media electrons is negligible, so the process can be treated as a positron annihilation with rested electrons and formulas [21] for cross section of electron-positron annihilation are applicable to this process. In contrast to the scaling with $\mathrm{Z}^{2}$ for the photon conversion in the field of the nucleus, we are dealing here with the interaction with atomic electrons, which scales with Z. Its cross section is small compared to the two-gamma annihilation (Fig. 9), however this reaction may be a source of background for different HEP experiments. The 


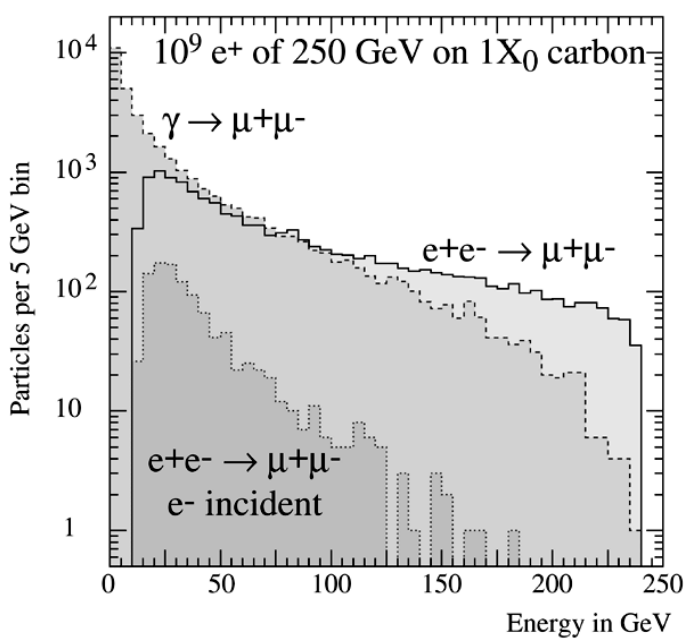

Fig. 10. Muon energy spectrum produced in the beam pipe of a linear collider after a carbon collimator of one radiation length thick. The $250 \mathrm{GeV}$ positron beam with $10^{9}$ particles per bunch is simulated.

Geant4 class G4AnnihiToMuPair has been implemented for sampling of the process.

\section{Positron Annihilation to Hadrons}

Electron-positron annihilation to hadrons has been studied for many years in $\mathrm{e}^{+} \mathrm{e}^{-}$colliders over a large range of energies and the cross sections involved are well known. A first implementation of positron annihilation to hadrons is now available in Geant4. This process is responsible for increasing of muon background for linear collider and has other similarity with the muon pair production. The threshold for the process is

$$
\mathrm{E}_{\mathrm{th}}=2 \mathrm{~m}_{\pi}^{2} / \mathrm{m}_{\mathrm{e}}-\mathrm{m}_{\mathrm{e}}=70.35 \mathrm{GeV} .
$$

In the first Geant 4 implementation of hadron production by positron, only pion pair production is simulated. The validity range of the model is limited to $1 \mathrm{TeV}$. For these energies the following reaction dominates:

$$
\mathrm{e}^{+} \mathrm{e}^{-} \rightarrow \gamma \rho \rightarrow \pi^{+} \pi^{-} \gamma
$$

where $\gamma$ is a radiative photon, which is emitted by the initial electron or positron. This radiative correction is essential, because it significantly modifies the shape of the resonance cross section of the $\rho$-meson (Fig. 9). Details of the theory used are described in [22], in which the main terms and the leading $\alpha^{2}$ corrections for this reaction are taken into account. The Geant 4 class G4eeToHadrons has been implemented for sampling of the process. It is foreseen to extend this process including production of other light vector mesons and nonresonant background for higher energies.

\section{Background in Linear Colliders}

Muon production in high energy electromagnetic showers can be a major source of background for high energy linear colliders (Fig. 10). From initially $10^{10}$ particles in the beam, a fraction of $10^{-3}$ may hit collimators and produce thousands of muons which could travel over several kilometers through the vacuum chamber and reach the detector region. The process of positron annihilation to hadrons provides hadron background in the beam pipe. It also increases the number of background muons because of pion decay. A good simulation is essential for the design of the collimation system, the final focus region and the detectors.

Several stages of collimation inside a linac will be necessary. High Z-materials like lead or tungsten would easily be destroyed under primary beam impact. For the first stage of collimation, relatively thin spoilers made of carbon are considered. For carbon $(\mathrm{Z}=6)$, the muon production by gamma conversion and positron annihilation will be comparable (Fig. 10). The positron annihilation is the dominant source of high energy muons, close to the positron energy. For an electron beam, positron annihilation only plays a minor role as a secondary process in the electromagnetic cascade in the production of lower energy muons.

\section{CONCLUSION}

The set of processes for muon production in media and muon interaction with media is available in the Geant 4 toolkit. The physics models implemented take main terms and leading corrections into account. The accuracy of these implementations is on the level of a few percent. The generally rather rare processes of positron annihilation to muon and pion pairs have been introduced. They are mainly important as a source of high energy muons and pions, which may be a background for LHC and linear collider experiments.

\section{ACKNOWLEDGMENT}

Authors are grateful to J. Apostolakis for valuable comments to the paper.

\section{REFERENCES}

[1] S. Agostinelli et al., Geant4-A simulation toolkit, in Nucl. Instr. Meth. A, vol. 506, pp. 250-303, 2003. Geant4 Collaboration.

[2] H. Burkhardt et al., "Geant4 standard electromagnetic package for HEP applications," in IEEE-NSS-33-179 Conference Record, 2004; MC'2005. Chattanuga, LaGrange Park, TN, Apr. 17-21, 2005. on CD-ROM, (2005).

[3] H. Burkhardt, V. M. Grichine, V. N. Ivanchenko, P. Gumplinger, R. P. Kokoulin, M. Maire, and L. Urbun. GEANT4 Standard Electromagnetic physics package. presented at Proc. MC2005

[4] R. P. Kokoulin and A. A. Petrukhin, "Theory of the pair meter for high energy muon measurements," Nucl. Instr. Meth. A, vol. 263, pp. 468-479, 1988.

[5] D. E. Groom, N. V. Mokhov, and S. I. Striganov, "Muon stopping power and range tables $10 \mathrm{MeV}-100 \mathrm{TeV}$," Atomic Data and Nuclear Data Tables, vol. 78, pp. 183-356, 2001.

[6] A. Allisy et al., "Stopping Powers and Ranges for Protons and Alpha Particles,", ICRU Rep. 49, 1993.

[7] S. R. Kelner, R. P. Kokoulin, and A. A. Petrukhin, "Bremsstrahlung from muons scattered by atomic electrons," Phys. Atomic Nuclei., vol. 60, pp. 576-583, 1997.

[8] K. Lassila-Perini and L. Urban, "Energy loss in thin layers in GEANT," Nucl. Instr. Meth., vol. A362, pp. 416-422, 1995.

[9] S. R. Kelner, R. P. Kokoulin, and A. A. Petrukhin, About Cross Section for High-Energy Muon Bremsstrahlung, Moscow, Russia, 1995. preprint MEPhI 024-95, CERN SCAN-9 510048.

[10] A. A. Petrukhin and V. V. Shestakov, "The influence of the nuclear and atomic form factors on the muon bremsstrahlung cross-section," Canad. J. Phys., vol. 46, pp. S377-S380, 1968. 
[11] R. P. Kokoulin and A. A. Petrukhin, "Analysis of the cross-section of direct pair production by fast muons," Acta. Phys. Acad. Sci. Hung., vol. 29, pp. 277-284, 1970. Supp. 1.4.

[12] electron pair production by high energy muons," Proc. 12th Int. Conf. Cosmic Rays, vol. 6, pp. 2436-2444, 1971.

[13] S. R. Kelner, "Pair production in collisions between muons and atomic electrons," Phys. Atomic Nuclei, vol. 61, pp. 448-456, 1998.

[14] V. V. Borog and A. A. Petrukhin, "The cross section of the nuclear interaction of high energy muons," Proc. 14th Intern. Cosmic Ray Conf., vol. 6, pp. 1949-1954, 1975.

[15] S. J. Brodsky, F. E. Close, and J. F. Gunion, "Phenomenology of photon processes, vector dominance and crucial tests for parton models," Phys. Rev. D, vol. 6, pp. 177-189, 1972.

[16] D. O. Caldwell et al., "Measurement of shadowing in photon-nucleus total cross sections from 20 to $185 \mathrm{GeV}$," Phys. Rev. Lett., vol. 42, pp. 553-556, 1979.
[17] L. B. Bezrukov and E. V. Bugaev, Nucleon shadowing effects in photon nucleus interaction, in Yad. Fiz. (Sov. J. Nucl. Phys.), vol. 33, pp. 1195-1207, 1981. (in Russian).

[18] R. P. Kokoulin and A. A. Petrukhin, "Muon interactions and consequences in underground physics," in Ital. Phys. Soc., Conf. Proc., vol. 57, F. Giovanelli and G. Mannocchi, Eds., 1997, pp. 379-393. Vulcano Workshop 1996 Frontier Objects in Astrophysics and Particle Physics.

[19] H. Burkhardt, S. R. Kelner, and R. P. Kokoulin, "Monte Carlo generator for muon pair production," Preprint CERN-SL-2002-016-AP, Geneva, Switzerland, 2002.

[20] - "Production of Muon Pairs in Annihilation of High-Energy Positrons with Resting Electrons,", CERN-AB-2003-002-ABP, 2003.

[21] S. Eidelman et al., "The review of particle physics," Phys. Lett. B, vol. 592, no. 1,2004

[22] M. Benayoun, S. I. Eidelman, V. N. Ivanchenko, and Z. K. Silagadze, "Spectroscopy at B-factories using hard photon emission," Mod. Phys. Lett. A, vol. 14, pp. 2605-2613, 1999. 